\title{
Curvas de lactação de vacas holandesas do Estado do Paraná, Brasil
}

\author{
Lactation curves of Holstein cows in the state of Parana, Brazil
}

\section{Carla Forte Maiolino Molento ${ }^{1}$ Humberto Monardes $^{2}$ Newton Pohl Ribas ${ }^{3}$ Elliot Block ${ }^{4}$}

RESUMO

Dados do Programa de Análise de Rebanhos Leiteiros do Paraná (PARLPR - APCBRH), contendo 27029 lactações encerradas de 422 rebanhos, foram utilizados para a visualização das curvas de lactação. As curvas foram calculadas de acordo com três grupos de parição e seis niveis de produção. A produção no momento do pico aumenta à medida que aumenta o nível de produção do rebanho ou a ordem de parição. O declínio diário aumenta à medida que aumenta o nível de produção do rebanho, para animais de segunda ou mais lactações; para animais de primeira lactação, não se observou tendência para esta característica. As curvas e os valores de pico e persistência obtidos podem ser utilizados como instrumentos de manejo em fazendas leiteiras do Paraná.

Palavras-chave: Brasil, curvas de lactação, Paraná, vacas holandesas.

\section{ABSTRACT}

A data set from the dairy herd analysis system in the State of Parana, Brazil (PARLPR - APCBRH), containing 27029 lactation records from 422 herds, was used to plot lactation curves. Lactation curves were plotted according to three parity groups and six levels of production. Production at peak increases with increases in herd production level and with increases in lactation number. Daily decline increases with increases in herd production level, in the case of second or greater lactation; for first lactation animals, no trend was identified for this characteristic. The curves, peak and persistency values obtained are to be used as management tools on dairy farms in the State of Parana.
Key words: Brazil, holstein cows, lactation curves, Parana.

\section{INTRODUÇÃO}

A Associação Paranaense de Criadores de Bovinos da Raça Holandesa (APCBRH) foi fundada em 1953. Treze anos mais tarde, a APCBRH estabeleceu o serviço de análise de rebanhos leiteiros, trabalhando com 88 vacas provenientes de três rebanhos. Hoje denominado Programa de Análise de Rebanhos Leiteiros do Paraná (PARLPR), este serviço havia acumulado um banco de dados de aproximadamente 140.000 lactações completas de 75.000 animais no ano de 1995. Apesar de apenas cerca de 3\% das vacas leiteiras do Estado encontrarem-se inscritas no PARLPR (RIBAS, 1989), os dados disponíveis são a melhor fonte de informação sobre a produção de leite local, assim como a respeito da qualidade do leite produzido, em termos de porcentuais de gordura e proteína e de contagem de células somáticas (CCS) (RIBAS, 1992).

As curvas de lactação e os parâmetros calculados a partir delas, pico e persistência da lactação, vêm sendo utilizados há muito tempo para auxiliar o manejo de fazendas leiteiras. A persistência da lactação foi definida na terceira década do século passado (GAINES, 1927); logo após, tanto a

\footnotetext{
${ }^{1}$ Médico Veterinário, MSc, PhD. Laboratório de Bem-Estar Animal (LABEA), R. Nery Kurtz, 380, 97110-520, Santa Maria, RS. E-mail: carlamolento@yahoo.com. Autor para correspondência.

${ }^{2}$ Engenheiro Agrícola , MSc, PhD, Professor, MacDonald Campus, McGill University, Ste. Annne de Bellevue, Quebec, Canada. ${ }^{3}$ Médico Veterinário, MSc, Professor, Departmento de Zootecnia, Universidade Federal do Paraná, Diretor Geral da Secretaria de Estado do Abastecimento e da Agricultura (SEAB).

${ }^{4}$ Senior Principal Research Scientist, MSc, PhD, Arm and Hammer Animal Nutrition Group, Church and Dwight Co., Inc., Princeton, New Jersey, USA.
} 
persistência quanto o pico de lactação foram relatados como as principais características para se descrever a curva de lactação (SANDERS, 1930). Mais tarde, surgiram modelos matemáticos tentando descrever a curva de lactação, sendo o modelo proposto por WOOD (1967) amplamente utilizado. Entretanto, alguns pesquisadores (KELLOG, 1977; SHANKS, 1981) afirmam que o modelo matemático mais adequado será diferente para cada região geográfica e para situação climática. Apesar da maior área do Brasil encontrar-se entre o Equador e o Trópico de Capricórnio, o Estado do Paraná apresenta um clima subtropical. De acordo com a classificação de Koeppen, o clima do Paraná é descrito como $\mathrm{Cfb}$ e $\mathrm{Cfa}$, que indicam regiões subtropicais úmidas mesotérmicas (IAPAR, 1978). Não se sabe qual o modelo matemático mais adequado para as curvas de lactação do rebanho leiteiro do Estado do Paraná. Além das dificuldades na escolha do modelo matemático para se estimar as curvas de lactação, os dados mensais de lactações encerradas da APCBRH nunca foram organizados para uma observação das curvas de lactação. A visualização das curvas reais, no sentido de se utilizar médias não ajustadas, é de grande interesse para propósitos de manejo, uma vez que a variação fisiológica da produção de leite ao longo de uma lactação pode confundir técnicos no que tange à expectativa de produção para um dado animal em determinado estágio de lactação. Pretendese fornecer, com as curvas médias reais dos animais no estado do Paraná, a expectativa de produção para os animais de acordo com o nível de produção do rebanho e a ordem de parição. Consultores técnicos devem ter este conhecimento para diagnosticar corretamente a situação produtiva de um rebanho leiteiro, localizar as áreas prioritárias de ação e prováveis soluções.

Neste trabalho, curvas de lactação foram construídas para que os produtores de leite se possam beneficiar do banco de dados da APCBRH através da aplicação das mesmas no manejo de rotina de suas fazendas. Os objetivos principais foram (1) visualizar as curvas de lactação de vacas holandesas do Estado do Paraná, (2) comparar as curvas obtidas com curvas reais e estimadas existentes na literatura, e (3) calcular parâmetros facilmente utilizáveis por produtores e técnicos de campo, tais como valores de pico e persistência da lactação.

\section{MATERIAL E MÉTODOS}

O banco de dados utilizado continha inicialmente 38104 lactações encerradas, de janeiro de 1992 a julho de 1995. As restrições impostas ao banco de dados diminuíram o número de lactações encerradas para 27029, distribuídas em 422 rebanhos, excluindose animais de outras raças, lactações com dados incompletos de procedência geográfica, animais com menos de 22 meses ou mais de 140 meses de idade, rebanhos com menos de 10 lactações encerradas e lactações com menos de 200 dias ou mais de 400 dias.

As curvas de lactação foram produzidas de acordo com a ordem de parição e com a produção média de leite aos primeiros 305 dias de lactação. Foram adotados três grupos de ordem de parição: (1) primeira, (2) segunda e (3) terceira ou mais lactações. A média de produção foi calculada somando-se a produção das lactações encerradas de todos os animais de um mesmo rebanho e dividindo-se esta somatória pelo número de lactações do rebanho em questão. $\mathrm{Na}$ sequiência, os rebanhos foram agrupados de acordo com suas médias de produção em seis níveis, correspondendo a menos de $6000 \mathrm{~kg}$ de leite por 305 dias, mais de $8000 \mathrm{~kg}$ de leite por 305 dias, e níveis intermediários com um incremento de $500 \mathrm{~kg}$ de leite. $\mathrm{O}$ eixo $y$ das curvas de lactação representa as médias de produção de leite para cada controle leiteiro (eixo $x$ ), respeitando-se os grupos de parição e os níveis de produção dos rebanhos.

O pico foi definido como o controle leiteiro de mais alta média de produção de leite para uma dada ordem de parição e um nível de produção dos rebanhos. As razões entre picos de produção de leite dos grupos de diferentes ordens de parição foram calculadas. A persistência foi analisada de duas formas diferentes, com base na inclinação da curva (Pi) e como um porcentual da produção de leite do mês anterior (P\%). A Pi foi definida como um coeficiente de regressão linear, da forma descrita por GRAVERT \& BAPTIST (1976), adaptada para incluir somente a fase descendente da curva (controles 2 a 10). A P\% foi calculada multiplicando-se o valor de um controle por 100 e dividindo-se o resultado pelo valor do controle anterior. Todos os cálculos foram realizados através da utilização do pacote estatístico SAS (SAS, 1985).

\section{RESULTADOS E DISCUSSÃO}

As produções de leite, proteína e gordura, para um período de lactação de no máximo 305 dias, foram $6930+1852 \mathrm{~kg}, 215+57 \mathrm{~kg}$ e $227+62 \mathrm{~kg}$, respectivamente. O teor médio de proteína do leite foi 3,12 “ $0,20 \%$; o teor médio de gordura do leite foi 3,30 “ $0,45 \%$. Os valores destas características para cada nível de produção encontram-se na tabela 1 . O período de lactação médio foi de $309+44$ dias em lactação, e encontrou-se uma tendência de aumento no número 
Tabela 1 - Médias de dias em lactação (DEL), produção de leite, produção e teor de proteína e produção e teor de gordura para o Estado do Paraná, agrupados por nível de produção dos rebanhos, no período de 1990 a 1995.

\begin{tabular}{|c|c|c|c|c|c|c|c|}
\hline \multirow{2}{*}{ Item } & \multirow{2}{*}{$\begin{array}{l}\text { Estado do } \\
\text { Paraná }\end{array}$} & \multicolumn{6}{|c|}{ Nível de produção dos rebanhos ${ }^{1}$} \\
\hline & & 1 & 2 & 3 & 4 & 5 & 6 \\
\hline DEL & $309 \pm 44^{2}$ & $298 \pm 46$ & $306 \pm 44$ & $310 \pm 43$ & $312 \pm 43$ & $316 \pm 43$ & $317 \pm 42$ \\
\hline Leite, $\mathrm{kg} / 305 \mathrm{~d}$ & $6930 \pm 1852$ & $5219 \pm 1337$ & $6256 \pm 1277$ & $6748 \pm 1402$ & $7255 \pm 1444$ & $7728 \pm 1574$ & $8660 \pm 1740$ \\
\hline $\begin{array}{l}\text { Proteína, } \\
\text { kg/305 d }\end{array}$ & $215 \pm 57$ & $162 \pm 42$ & $194 \pm 40$ & $212 \pm 43$ & $226 \pm 44$ & $240 \pm 47$ & $268 \pm 52$ \\
\hline Proteína, \% & $3,12 \pm 0,20$ & $3,11 \pm 0,21$ & $3,11 \pm 0,20$ & $3,15 \pm 0,20$ & $3,12 \pm 0,19$ & $3,12 \pm 0,19$ & $3,10 \pm 0,18$ \\
\hline $\begin{array}{l}\text { Gordura, } \\
\text { kg/305 d }\end{array}$ & $227 \pm 62$ & $177 \pm 48$ & $212 \pm 47$ & $222 \pm 51$ & $235 \pm 52$ & $252 \pm 58$ & $273 \pm 63$ \\
\hline Gordura, \% & $3,30 \pm 0,45$ & $3,41 \pm 0,41$ & $3,39 \pm 0,40$ & $3,30 \pm 0,45$ & $3,26 \pm 0,44$ & $3,27 \pm 0,47$ & $3,18 \pm 0,51$ \\
\hline
\end{tabular}

${ }^{1}$ Nível de produção dos rebanhos: 1 = até $<6000 \mathrm{~kg}$ de leite; $2=$ de $6000 \mathrm{a}<6500 \mathrm{~kg}$ de leite; $3=$ de $6500 \mathrm{a}<7000 \mathrm{~kg}$ de leite; $4=$ de 7000 a $<7500 \mathrm{~kg}$ de leite; $5=$ de 7500 a $<8000 \mathrm{~kg}$ de leite; $6=8000 \mathrm{~kg}$ de leite ou mais. Somente a produção de leite até os $305 \mathrm{~d}$ de lactação foi considerada.

${ }^{2}$ Médias reais \pm desvio padrão.

de dias em lactação à medida que aumentou o nível de produção do rebanho (Tabela 1). Esta tendência não afetou o cálculo do nível de produção dos rebanhos, pois, para esse propósito, utilizou-se dados de, no máximo, 305 dias em lactação. O número de lactações encerradas parece estar distribuído de forma equilibrada entre os diferentes níveis de produção, em contraste com o número de rebanhos, que mostrou tendência de diminuição à medida que o nível de produção aumentou. (Tabela 2 ).

Nas figuras 1, 2 e 3 encontram-se as curvas de lactação médias para vacas em primeira, segunda e terceira ou mais lactações, respectivamente, sempre agrupadas de acordo com o nível de produção dos rebanhos de proveniência dos animais. As curvas são relativamente paralelas para todos os níveis de produção. As curvas foram mais altas e com inclinações mais pronunciadas à medida que a ordem de lactação aumentou. O pico de produção ocorreu invariavelmente durante o segundo controle. Sendo o intervalo médio entre controles 30,5 dias, é correto concluir que o pico de produção ocorreu durante o segundo mês de lactação. Estes resultados contrastam com VAZ DE OLIVEIRA et al. (2003), que não observaram fase ascendente e nem pico de lactação para as curvas de lactação de vacas F1 holandês-gir, criadas no Estado de Minas Gerais. Entretanto, as curvas de lactação de vacas holandesas de Minas Gerais também parecem apresentar um pico de lactação, pela observação das curvas estimadas por GONÇALVES et al. (2002). As curvas de lactação das vacas holandesas do Estado do Paraná mostram características similares àquelas das curvas de lactação da América do Norte (JONES, 1991; LEFEBVRE et al.,

Tabela 2 - Distribuição das lactações encerradas e dos rebanhos através dos diferentes níveis de produção, para o Estado do Paraná no período de 1990 a $1995^{1}$.

\begin{tabular}{lcccc}
\hline $\begin{array}{l}\text { Nível de produção } \\
\text { do rebanho }\end{array}$ & Lactações encerradas & $\begin{array}{c}\text { Porcentual do total de } \\
\text { lactações encerradas }\end{array}$ & $\begin{array}{c}\text { Rebanhos } \\
\text { rebanhos }\end{array}$ \\
\hline 1 & 5540 & $20.5 \%$ & 136 & $32.2 \%$ \\
2 & 3472 & $12.8 \%$ & 60 & $14.2 \%$ \\
3 & 4356 & $16.1 \%$ & 64 & $15.2 \%$ \\
4 & 5182 & $19.2 \%$ & 65 & $15.4 \%$ \\
5 & 4020 & $14.9 \%$ & 48 & $11.4 \%$ \\
6 & 4459 & $16.5 \%$ & 49 & $11.6 \%$ \\
Total & 27029 & $100.0 \%$ & 422 & $100.0 \%$ \\
\hline
\end{tabular}

${ }^{1}$ Nível de produção dos rebanhos: 1 = até $<6000 \mathrm{~kg}$ de leite; $2=$ de 6000 a $<6500 \mathrm{~kg}$ de leite; $3=$ de 6500 a $<7000 \mathrm{~kg}$ de leite; $4=$ de 7000 a $<7500 \mathrm{~kg}$ de leite; 5 = de 7500 a $<8000 \mathrm{~kg}$ de leite; $6=8000 \mathrm{~kg}$ de leite ou mais. Somente a produção de leite até os 305 d de lactação foi considerada. 


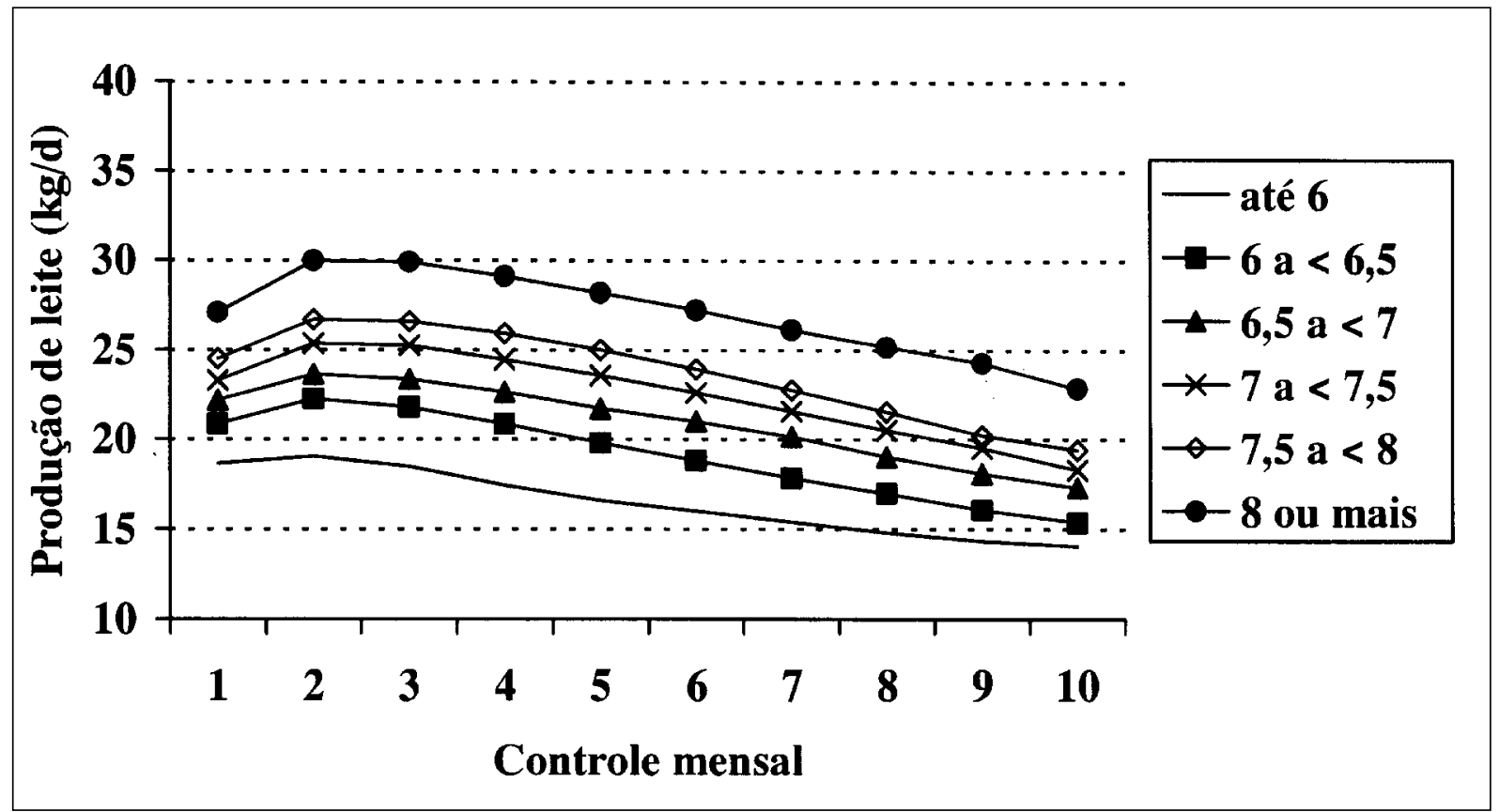

Figura 1 - Curvas de lactação para animais de primeira lactação, de acordo com o nível de produção dos rebanhos (em 1000 kg de leite/305 d), para o Estado do Paraná, no período de 1990 a 1995, sendo o dia da visita do supervisor de campo do PARLPR, com a anotação da produção de leite de cada animal, chamado de controle mensal e numerado em relação ao parto.

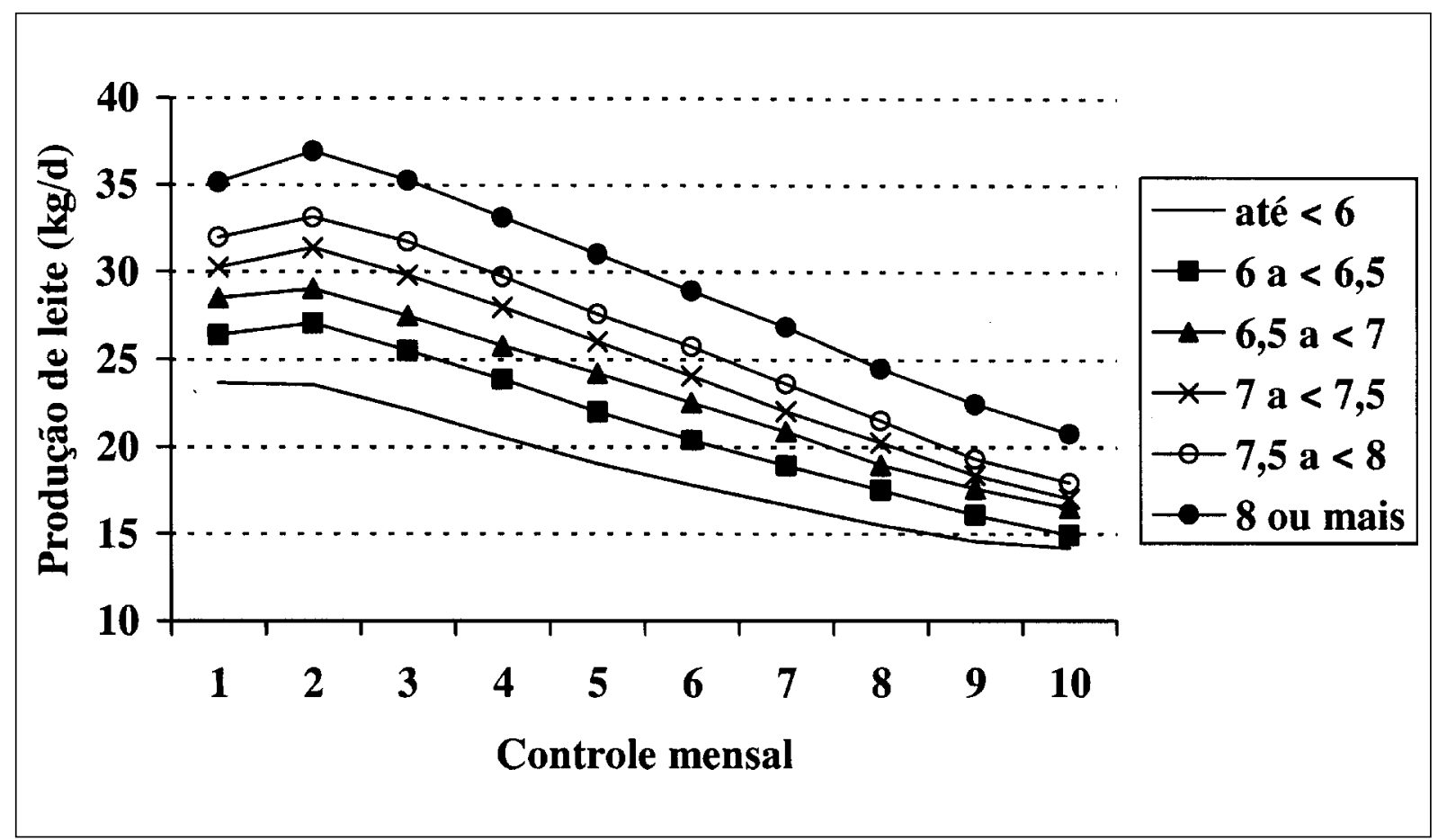

Figura 2 - Curvas de lactação para animais de segunda lactação, de acordo com o nível de produção dos rebanhos (em $1000 \mathrm{~kg}$ de leite/305 d), para o Estado do Paraná, no período de 1990 a 1995, sendo o dia da visita do supervisor de campo do PARLPR, com a anotação da produção de leite de cada animal, chamado de controle mensal e numerado em relação ao parto. 


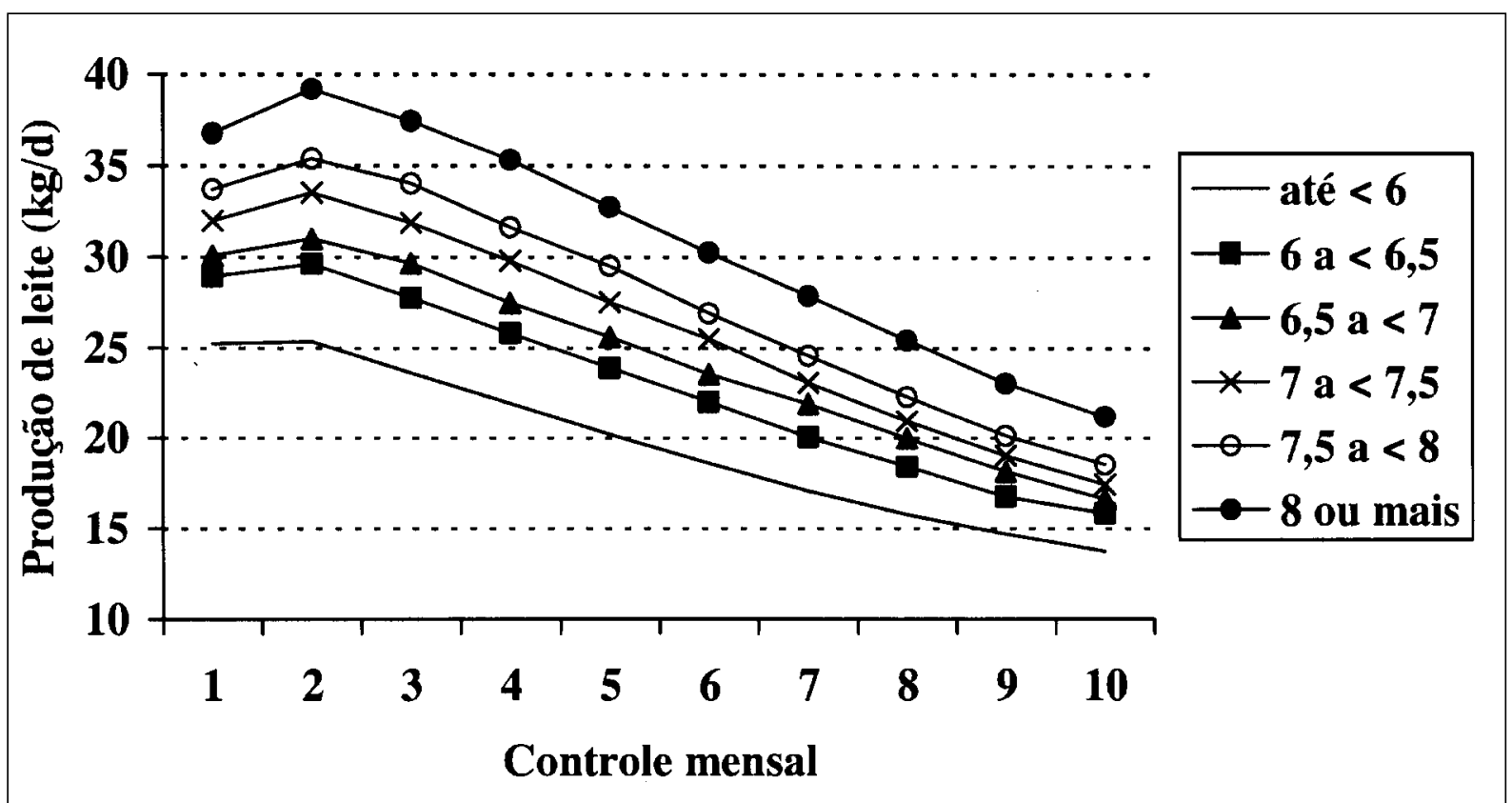

Figura 3 - Curvas de lactação para animais de terceira lactação ou mais, de acordo com o nível de produção dos rebanhos (em 1000 kg de leite/305 d), para o Estado do Paraná, no período de 1990 a 1995, sendo o dia da visita do supervisor de campo do PARLPR, com a anotação da produção de leite de cada animal, chamado de controle mensal e numerado em relação ao parto.

1995). O evidente pico de produção das curvas obtidas indica que modelos matemáticos com pontos de produção máxima, como o modelo clássico de WOOD (1967), são os melhores candidatos para descrever as curvas de lactação das vacas holandesas criadas no Estado do Paraná.

As produções médias no pico de lactação para as diferentes ordens de parição estão listadas na tabela 3. A produção de leite no pico de lactação foi diretamente proporcional à ordem de parição e ao nível de produção do rebanho. A razão entre os picos de animais de primeira e segunda lactação foi $81 \%$, segunda e terceira ou mais $93 \%$, e primeira e terceira ou mais $76 \%$; estas razões foram similares para todos os níveis de produção. As duas razões comparando dados de primeira lactação com os outros (primeira : segunda, primeira : terceira ou mais) foram maiores que as mesmas razões calculadas para o nordeste dos Estados Unidos (JONES, 1991). Provavelmente isto se explique por uma maior diferença de potencial genético entre vacas mais velhas e mais novas no Estado do Paraná em relação aos rebanhos americanos. Esta maior diferença significa que as vacas de primeira lactação em um rebanho brasileiro apresentam uma superioridade genética de maior amplitude em relação às vacas de vida produtiva mais avançada no mesmo rebanho, se comparada à superioridade genética dos mesmos animais em rebanhos norte-americanos.

A Pi é expressa em valores negativos de $\mathrm{kg}$ de leite por dia, representando o declínio diário médio, sendo todos os valores de Pi obtidos para as diferentes ordens de parição e os diferentes níveis de produção apresentados na tabela 3. Para animais de primeira lactação, a Pi não demonstrou nenhuma tendência entre os diferentes níveis de produção; desta forma, um declínio diário médio na produção de leite de $0,026 \mathrm{~kg} \mathrm{~d}^{-1}$ deve ser esperado para esta ordem de parição. No caso de animais de segunda e terceira ou mais lactações, a Pi demonstrou uma tendência de diminuição à medida que o nível de produção do rebanho aumentou; em outras palavras, o declínio diário em valores absolutos (kg de leite) é mais acentuado para rebanhos de maior nível de produção para vacas de duas ou mais lactações.

A P\% média de todos os níveis de produção, segundo os três grupos de ordem de parição, aparece na tabela 4 . Animais de primeira lactação apresentam uma $\mathrm{P} \%$ mais alta se comparados àqueles de segunda e terceira ou mais lactações. Animais de segunda lactação apresentam uma $\mathrm{P} \%$ levemente mais alta em relação aos animais de terceira ou mais lactações. A P\% tendeu a decrescer à medida que aumentava o número de dias em lactação, exceto para os últimos valores de 
Tabela 3 - Valores de pico de produção de leite e de persistência baseada na inclinação da curva (Pi), de acordo com a ordem de parição e o nível de produção dos rebanhos, para o Estado do Paraná no período de 1990 a 1995.

\begin{tabular}{|c|c|c|c|c|c|c|}
\hline \multirow{2}{*}{ Nível de produçãol ${ }^{1}$} & \multicolumn{2}{|c|}{ Primeira lactação } & \multicolumn{2}{|c|}{ Segunda lactação } & \multicolumn{2}{|c|}{ Terceira lactação ou mais } \\
\hline & Pico, kg/d & $\mathrm{Pi}, \mathrm{kg} / \mathrm{d}$ & Pico, kg/d & $\mathrm{Pi}, \mathrm{kg} / \mathrm{d}$ & Pico, kg/d & $\mathrm{Pi}, \mathrm{kg} / \mathrm{d}$ \\
\hline 1 & $19,0^{\mathrm{a}, 2}$ & $-0,026^{a}$ & $23,5^{\mathrm{a}}$ & $-0,042^{a}$ & $25,3^{\mathrm{a}}$ & $-0,053^{\mathrm{a}}$ \\
\hline 2 & $22,2^{\mathrm{b}}$ & $-0,029^{\mathrm{b}}$ & $27,1^{\mathrm{b}}$ & $-0,055^{\mathrm{b}}$ & $29,6^{b}$ & $-0,063^{\mathrm{c}}$ \\
\hline 3 & $23,6^{\mathrm{c}}$ & $-0,025^{\mathrm{a}}$ & $29,0^{\mathrm{c}}$ & $-0,056^{\mathrm{b}, \mathrm{c}}$ & $31,0^{\mathrm{c}}$ & $-0,061^{\mathrm{c}}$ \\
\hline 4 & $25,3^{\mathrm{d}}$ & $-0,024^{a}$ & $31,4^{\mathrm{d}}$ & $-0,060^{\mathrm{c}}$ & $33,5^{\mathrm{d}}$ & $-0,057^{\mathrm{b}}$ \\
\hline 5 & $26,7^{\mathrm{e}}$ & $-0,027^{\mathrm{a}, \mathrm{b}}$ & $33,1^{\mathrm{e}}$ & $-0,064^{\mathrm{d}}$ & $35,4^{\mathrm{e}}$ & $-0,072^{\mathrm{d}}$ \\
\hline 6 & $30,0^{\mathrm{f}}$ & $-0,026^{a, b}$ & $37,0^{\mathrm{f}}$ & $-0,065^{\mathrm{d}}$ & $39,2^{\mathrm{f}}$ & $-0,074^{\mathrm{d}}$ \\
\hline
\end{tabular}

${ }^{1}$ Nível de produção dos rebanhos: 1 = até $<6000 \mathrm{~kg}$ de leite; $2=$ de 6000 a $<6500 \mathrm{~kg}$ de leite; $3=$ de 6500 a $<7000 \mathrm{~kg}$ de leite; $4=$ de 7000 a $<7500 \mathrm{~kg}$ de leite; 5 = de 7500 a $<8000 \mathrm{~kg}$ de leite; $6=8000 \mathrm{~kg}$ de leite ou mais. Somente a produção de leite até os $305 \mathrm{~d}$ de lactação foi considerada.

${ }^{2}$ Valores na mesma coluna com letras diferentes diferem estatisticamente $(P<0,01$, teste $t)$.

animais de segunda e terceira ou mais lactações. Esta exceção foi devido aos animais que terminaram a lactação antes dos 305 dias, desta forma não entrando no cálculo da produção média de leite dos últimos controles. Uma vez que estes animais provavelmente produziam menos do que a média, o efeito dos mesmos não participarem nas últimas médias de $\mathrm{P} \%$ foi uma aparência de valores mais altos para as mesmas. Assim, esta característica é um artefato da distribuição do período de lactação em relação aos 305 dias, não se tratando de uma característica fisiológica da lactação das vacas holandesas no Estado do Paraná. Não se deve ter a

Tabela 4 - Persistências porcentuais ( $\mathrm{P} \%$ ) médias para todos os níveis de produção, de acordo com o número de lactação, para o Estado do Paraná, no período de 1990 a 1995, sendo o dia da visita do supervisor de campo do PARLPR, com a anotação da produção de leite de cada animal, chamado de controle mensal e numerado em relação ao parto.

\begin{tabular}{lccc}
\hline & \multicolumn{3}{c}{ Persistência porcentual (P\%) } \\
\cline { 2 - 4 } Controle \\
\cline { 2 - 4 } mensal & $\begin{array}{c}\text { Primeira } \\
\text { lactação }\end{array}$ & $\begin{array}{c}\text { Segunda } \\
\text { lactação }\end{array}$ & $\begin{array}{c}\text { Terceira lactação } \\
\text { ou mais }\end{array}$ \\
\hline $2: 1$ & $107,3 \%$ & $102,7 \%$ & $103,7 \%$ \\
$3: 2$ & $98,7 \%$ & $94,8 \%$ & $94,9 \%$ \\
$4: 3$ & $96,4 \%$ & $93,6 \%$ & $93,2 \%$ \\
$5: 4$ & $95,9 \%$ & $93,0 \%$ & $92,7 \%$ \\
$6: 5$ & $96,0 \%$ & $93,0 \%$ & $92,0 \%$ \\
$7: 6$ & $95,6 \%$ & $92,5 \%$ & $91,6 \%$ \\
$8: 7$ & $95,2 \%$ & $91,7 \%$ & $91,3 \%$ \\
$9: 8$ & $95,3 \%$ & $91,8 \%$ & $91,0 \%$ \\
$10: 9$ & $95,5 \%$ & $93,7 \%$ & $92,6 \%$ \\
\hline
\end{tabular}

expectativa de maior $\mathrm{P} \%$ no final da lactação ao se trabalhar com curvas de lactação individuais.

\section{CONCLUSÃO}

Por meio das curvas de lactação de vacas holandesas inscritas no PARLPR, observa-se que as características de produção de leite à medida que a lactação avança seguem as mesmas tendências que as curvas do nordeste dos Estados Unidos (JONES, 1991) e da província de Quebec, Canadá (LEFEBVRE et al., 1995). Os valores médios obtidos para o pico de produção, Pi (declínio diário) e P\% prestam-se para utilização como instrumentos de manejo com vários objetivos, pois dão ao técnico de campo uma noção da expectativa de produção de leite em todas as fases da lactação. Assim, pode-se diagnosticar a situação produtiva de um rebanho, localizar áreas prioritárias de ação e possíveis soluções.

\section{AGRADECIMENTOS}

Os autores agradecem a Clotilde de L. B. Germiniani e Rodrigo de Almeida (ambos da Universidade Federal do Paraná) e Roger Cue (McGill University, Quebec, Canadá) pela ajuda essencial ao desenvolvimento dos trabalhos. A indispensável e gentil cessão dos dados pela Associação Paranaense de Criadores de Bovinos Raça Holandesa (APCBRH) é reconhecida. Este trabalho foi financiado pelo Conselho de Aperfeiçoamento de Pessoal de Ensino Superior (CAPES) e pela Canadian International Development Agency (CIDA).

\section{REFERÊNCIAS BIBLIOGRÁFICAS}

GAINES, W.L. Persistency of lactation in dairy cows. Ill Agric Exp Stn. Bull, v.288, p.15-20, 1927. 
GONÇALVES, T.M. et al. Curvas de lactação em rebanhos da raça holandesa no Estado de Minas Gerais. Escolha do modelo de melhor ajuste. R Bras Zootec, v.31, p.16891694, 2002.

GRAVERT, H.O.; BAPTIST, R. Breeding for persistency of milk yield. Livestock Production Science, v.3, p.27-31, 1976.

IAPAR - Instituto Agronômico do Paraná. Cartas climáticas básicas do Estado do Paraná. Londrina : IAPAR, 1978. $41 \mathrm{p}$

JONES, L.R. Lactation curves for monitoring milk production. In: Dairy Science Handbook. Texas: International Stockman's School, Houston, 1991. V.20.

KELLOG, D.W.; URQUHART, N.S.; ORTEGA, A.J. Estimating Holstein lactation curves with a gamma curve. J Dairy Sci, v.60, p.1303-1315, 1977.

LEFEBVRE, D. et al. La gestion de la performance du troupeau laitier: des outils à exploiter. In: SYMPOSIUM SUR LES BOVINS LAITIERS, 19., 1995. Cahier de Conférence. Montreal : Conseil des productions animales du Québec Inc., 1995. p.13-56.
RIBAS, N.P. Serviço de controle leiteiro no Paraná. Revista Gado Holandês, v.161, p.13-24, 1989.

RIBAS, N.P. Controle leiteiro no Brasil - Laboratório Centralizado de Análise de Leite do Paraná. Revista Gado Holandês, v.402, p.10, 1992.

SANDERS, H.G. The analysis of the lactation curve into maximum yield and persistency. J Agric Sci Camb, v.20, p.145-185, 1930 .

SAS INSTITUTE. SAS User's Guide: Statistics. Version 6 ed. Cary, NC. : SAS Inst, 1985. 95p.

SHANKS, R.D. et al. Genetic aspects of lactation curves. J Dairy Sci, v. 64, p.1852-1860, 1981.

VAZ DE OLIVEIRA, H.T. et al. Estudo da curva da lactação, ajustada pela função gama incompleta, de vacas F1 holandêsgir. In: REUNIÃO ANUAL DA SOCIEDADE BRASILEIRA DE ZOOTECNIA , 40., 2003, Santa Maria, RS. Anais... Santa Maria, 2003. CD-ROM.

WOOD, P.D.P. Algebraic model of the lactation curve in cattle. Nature, v.218, p.894, 1967. 